\title{
Spinal cord regeneration: A brief overview of present scenario and a sneak peek into the future
}

\author{
Ekta Srivastava $^{1}$, Anamika Singh ${ }^{1}$, and Ashok Kumar ${ }^{2}$ \\ ${ }^{1}$ Indian Institute of Technology Kanpur \\ ${ }^{2}$ Indian Institute of Technology
}

March 9, 2021

\begin{abstract}
Central nervous system (CNS) portrays appreciable complexity in developing from a neural tube to controlling major functions of the body and orchestrated co-ordination in maintaining its homeostasis. Any insult or pathology to such an organized tissue leads to a plethora of events ranging from local hypoxia, ischemia, oxidative stress to reactive gliosis and scarring. Despite unravelling the pathophysiology of spinal cord injury (SCI) and linked cellular and molecular mechanism, the over exhaustive inflammatory response at the site of injury, limited intrinsic regeneration capability of CNS, and the dual role of glial scar halts the expected accomplishment. The review discusses major current treatment approaches for traumatic SCI, addressing their limitation and scope for further development in the field under three main categories- neuroprotection, neuro-regeneration, and neuroplasticity. We further propose that a multi-disciplinary combinatorial treatment approach exploring any two or all three heads simultaneously could alleviate the inhibitory milieu and ameliorate functional recovery.
\end{abstract}

\section{Hosted file}

Srivastava et al._Review Article_09-03-2021.pdf available at https://authorea.com/users/ 400470/articles/512799-spinal-cord-regeneration-a-brief-overview-of-present-scenarioand-a-sneak-peek-into-the-future 